\title{
Risk of T1DM increases with early and late weaning
}

The exact timing of first exposure to solid food increases the risk of developing type 1 diabetes mellitus (T1DM) in infants with a genetically increased risk of the disease, suggests a prospective study from the USA. Both early ( $<4$ months of age) and late ( $\geq 6$ months of age) first exposure to solids predicted development of T1DM.

The incidence of T1DM is increasing worldwide, with the most rapid increase being reported for children aged $<5$ years. Infant diet exposures, including breastmilk, cow's milk and various solid foods, such as gluten, rice, fruit and root vegetables, have previously been explored as candidate risk factors for T1DM, but results from retrospective studies have been conflicting about any effect on diabetes or autoimmunity risk.

In a prospective, observational study, the researchers analysed the association between infant diet exposures and the development of T1DM in 1,835 children from the DAISY (Diabetes Autoimmunity Study in the Young) cohort. These children had previously been determined to have an increased genetic risk of T1DM (owing to HLA genotype). Over time, a total of 53 children developed T1DM. "A prospective study is necessary for this type of question because the exposures of interest occur very early in the child's life," explains Jill Norris (University of Colorado), senior author of the study. "Given that the average age of T1DM onset is $~ 10$ years, it is improbable that mothers would be able to accurately remember their child's diet during infancy, which makes a retrospective study problematic." Intriguingly, the investigators found that if mothers were breastfeeding at the time when gluten-containing foods were introduced to the baby, the risk of T1DM was reduced. "This result is similar to a finding from a Swedish study that found that breastfeeding while introducing gluten-containing foods might reduce the risk of coeliac disease, an autoimmune condition that has several similarities with T1DM," says Norris.

Taken together, the results suggest that the safest age to introduce solid foods in

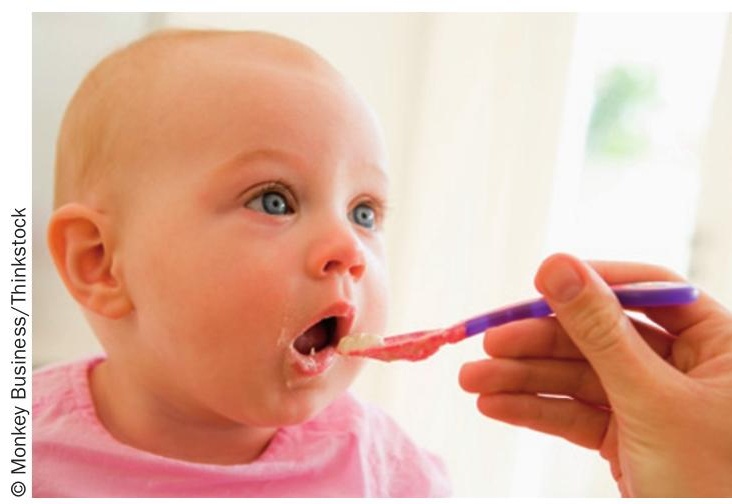

children at increased genetic risk of T1DM is between 4 months and 5 months of age, and breastfeeding while introducing new foods might reduce T1DM risk.

Linda Koch

Original article Frederiksen, B. et al. Infant exposures and development of type 1 diabetes mellitus-The Diabetes Autoimmunity Study in the Young (DAISY). JAMA Pediatr. doi:10.1001/jamapediatrics.2013.317 